https://doi.org/10.15407/ujpe63.8.759

I. OVSIIENKO,${ }^{1}$ T. LEN,${ }^{1}$ L. MATZUI, ${ }^{1}$ O. LAZARENKO, ${ }^{1}$ F. LE NORMAND, ${ }^{2}$ A. SHAMES ${ }^{3}$

${ }^{1}$ Physical Department, Taras Shevchenko National University of Kyiv (2, Prosp. Academician Glushkov, Kyiv 03022, Ukraine; e-mail: intercalant@univ.kiev.ua)

2 Institut de Physique et Chimie des Matériaux (23, Rue du Loess BP 43, 67037 Strasbourg, France)

3 Department of Physics, Ben-Gurion University of the Negev (Be'er-Sheva, Israel)

\title{
FEATURES OF MICROSTRUCTURE OF CHEMICALLY OBTAINED GRAPHENE-LIKE PARTICLES ${ }^{1}$
}

\begin{abstract}
The graphene-like structures are investigated by methods of electron microscopy, EMR, and Raman spectroscopy. They were obtained by the chemical treatment and the sonication in different reagents. As a source for obtaining the graphene-like structures, the thermoexfoliated graphite was used. The number of graphite layers in the graphene-like structures, the shapes of individual particles, structural-morphological characteristics, and the homogeneity of the sizes of particles for specimens of the graphene-like structures obtained with different methods are estimated.
\end{abstract}

Keywords: graphene-like structures, chemical treatment, thermoexfoliated graphite, Raman spectroscopy.

\section{Introduction}

Significant interest to graphene is due to the spectrum of its unique electrophysical, electrodynamic, mechanical, and optical properties. Graphene is characterized by extremely high electrical conductivity [1]. The conductivity of graphene has a ballistic character that is caused by extremely high values of charge carrier's mobility even in comparison with single-wall carbon nanotubes (CNTs) and monocrystalline graphite [2]. For graphene and graphene-like structures, there are a number of quantum effects, in particular, the quantum Hall effect and quantum linear magnetoresistance [3]. Graphene, as well as CNTs, is considered as a basis for the creation of future nanoelectronic devices [4]. Moreover, graphene is characterized by the high mechanical stiffness, thermal conductivity, and specific surface, the value of

(C) I. OVSIIENKO, T. LEN, L. MATZUI, O. LAZARENKO, F. LE NORMAND, A. SHAMES, 2018

ISSN 2071-0194. Ukr. J. Phys. 2018. Vol. 63, No. 8 which is even slightly higher than for CNTs $[5,6$, 7]. All these properties in combination with high electrical conductivity determine the prospect of using graphene and graphene-like structures as fillers for polymeric composites $[8,9]$. One of the most rapidly developing areas is the creation of polymer composites with graphene-like fillers for the use for electromagnetic shielding [10].

One of the priority tasks of modern nanotechnologies is the development of methods that would allow graphene-like structures (GLSs) with a high degree of structural perfection to be obtained in significant quantities. One of the most productive methods to obtain GLSs is the chemical treatment of layer carbon materials by strong oxidizers with the following dispersion. Usually, sonication is used for

1 The paper was presented at the XXIII Galyna Puchkovska International School-Seminar "Spectroscopy of Molecules and Crystals". 
Table 1. Methods to produce specimens of GLSs

\begin{tabular}{|c|c|c|}
\hline $\begin{array}{l}\text { Method and } \\
\text { corresponding } \\
\text { specimen No. }\end{array}$ & Reagent & Stages of treatment \\
\hline No. 1 & $1.5 \mathrm{M}$ solution of $\mathrm{KMnO}_{4}$ in $\mathrm{H}_{2} \mathrm{SO}_{4}$ & $\begin{array}{l}\text { 1) Preparing a suspension of TEG in the corresponding reagent. } \\
\text { Dispersion of the suspension in a magnetic stirrer for } 1 \mathrm{~h}\end{array}$ \\
\hline No. 2 & Mixture of $\mathrm{H}_{2} \mathrm{SO}_{4}(95 \%)$ and $\mathrm{HNO}_{3}$ & 2) Impregnation for $24 \mathrm{~h}$ \\
\hline No. 3 & $\begin{array}{l}(65 \%), \text { volume ratio is } 3: 1 \\
\text { Mixture of } 62.5 \% \text { vol.fr. ethanol } \\
\text { and } 37.5 \% \text { vol.fr. toluene }\end{array}$ & $\begin{array}{l}\text { 3) Repeated dispersion of the suspension in a magnetic stirrer for } 1 \mathrm{~h} \\
\text { 4) Filtration and washing with a distilled deionized water to remove } \\
\text { a sediment until neutral } \mathrm{pH}\end{array}$ \\
\hline No. 4 & Ethanol & 5) Drying a powder at $390 \mathrm{~K}$ up to a constant mass ( $8 \mathrm{~h}$ ) \\
\hline No. 5 & Ethanol & $\begin{array}{l}\text { 1. Sonication of TEG in the corresponding medium during } 1.5 \mathrm{~h} \\
(\text { No. } 5), 3 \mathrm{~h} \text { (No. } 6) \text { and } 20 \mathrm{~h} \text { (No. } 7)\end{array}$ \\
\hline No. 6 & Acetone & 2. Drying a powder at $390 \mathrm{~K}$ up to a constant mass \\
\hline No. 7 & Water & \\
\hline
\end{tabular}

the dispersing. The treatment of a source graphite by strong oxidizers, on the one hand, leads to the destruction of a lamellar graphite structure and the formation of graphene-like sheets. But, on the other hand, it stimulates the appearance of a significant number of defects in graphite layers. The chemical treatment of graphite with strong oxidizers results in the destruction of the delocalized $\pi$-electron system of a graphite layer and in the partial $\sigma$-bond rupture in the layer. This causes the deterioration of electrical transport properties of obtained graphenelike structures [11]. Thus, it is necessary to select optimum chemical reagents and methods of dispersion to obtain GLS with a small number of imperfect graphite layers.

The work presents the results of studies of a change in the surface morphology of nanocarbon particles and in a microstructure after the chemical treatment with strong oxidizers and after the sonication.

\section{Experimental}

For obtaining a GLS, thermoexfoliated graphite (TEG) was used as a source. The detailed description of the method to produce TEG is presented in [12].

Table 1 presents the methods that were used to obtain chemically treated specimens (Nos. 1-4) and sonicated specimens (Nos. 5-7) of GLS.

As is seen from the table, the strong unorganic and organic oxidizers were used in order to obtain GLSs. In addition, for comparison, the specimens were pre- pared by the sonication of source TEG in different media.

The surface morphology and microstructure of GLSs after the chemical treatment and the sonication were investigated with electron microscopy including high-resolution electron microscopy (electron microscope JEOL 200). The EMR method was used for the analysis of the order degree and defects in graphite layers. Room-temperature EMR measurements were conducted with the use of a Bruker EMX-220 X-band $(\nu \sim 9.4 \mathrm{GHz})$ spectrometer equipped with an $\mathrm{Ox}-$ ford Instrument ESR900 and/or a Bruker ER 4121 VT. For the determination of the deficiency degree of graphene-like sheets, Raman spectroscopy was used (Horiba LabRAM ARAMIS Raman spectrometer).

\section{Results and Discussion}

Figure 1 presents the fragments of SEM- and TEMimages of the GLSs obtained by the treatment with potassium permanganate in a sulfuric acid solution (No. 1) and with a mixture of sulfuric and nitric acids (No. 2).

As it follows from the presented figures, the shape and the edge state of GLSs are different for specimens obtained with different agents. In a case of GLS treated according to method No. 1, the elongated particles with a distinctive smooth edge are observed. In a case of GLSs obtained by method No. 2, the particles of rounded shape and partially ragged edges are observed. The linear defects are visualized on the GLS surface treated by both methods as plane faults,

ISSN 2071-0194. Ukr. J. Phys. 2018. Vol. 63, No. 8 


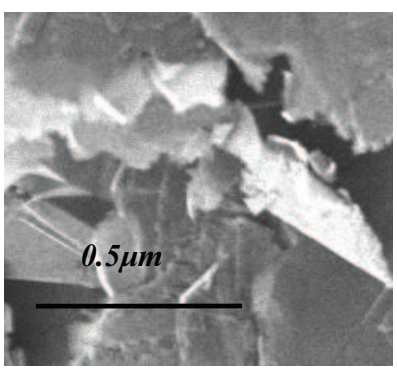

$a$

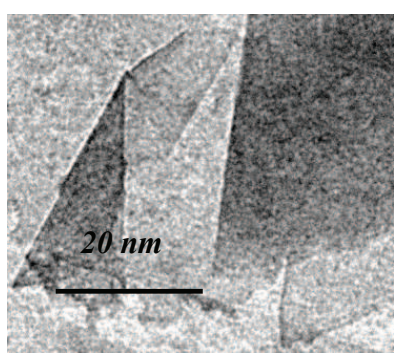

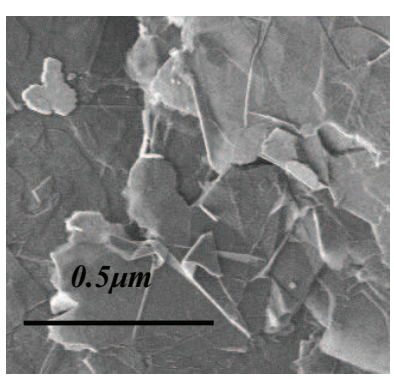

$b$

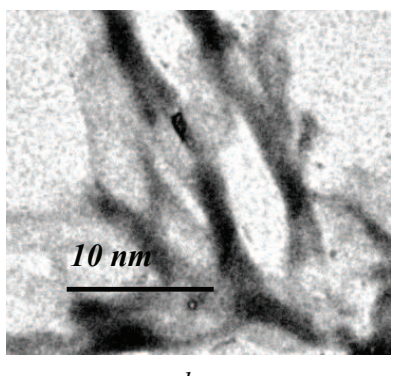

Fig. 1. Fragments of SEM $(a, b)$ and TEM $(c, d)$-images of nanographite platelets obtained with methods No. $1(a, c)$ and No. $2(b, d)$

lines of twinning, and outcrop of inner planes. The essential difference between particles' shapes treated with different methods is observed also on the TEM images. Separate particles of GLSs treated with using the solution of potassium permanganate in sulfuric acid have the shape of flat scales with smooth edges. Scales sizes $\sim 80 \mathrm{~nm}$. Scales can be bended twice along the lines of defects, and the bending line remains straight. Another kind of a scale shape is observed for GLSs treated with method No. 2. Flakes have twisted ribbon structure with the width less than $40 \mathrm{~nm}$ and the length less than $100 \mathrm{~nm}$. Thus, there is a direct relation between the chemical agent and the structural-morphological state of obtained GLSs.

To determine the degree of structural ordering in GLSs and to reveal carbon defects in graphite planes (dangling bonds), the EMR investigation of the obtained GLSs was carried out.

Figure 3 presents the RTEMR spectrum of a specimen treated with a mixture of sulfuric and nitric acids (No. 2): full spectrum (a) and the region in the vicinity of $g=2.00(b)$.

Evidently, this specimen absorbs the electric component of MW less than specimen 1. For this spec-

ISSN 2071-0194. Ukr. J. Phys. 2018. Vol. 63, No. 8

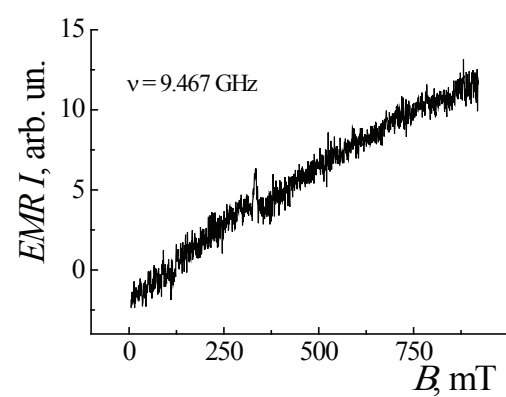

Fig. 2. RTEMR spectrum of specimen No. 1
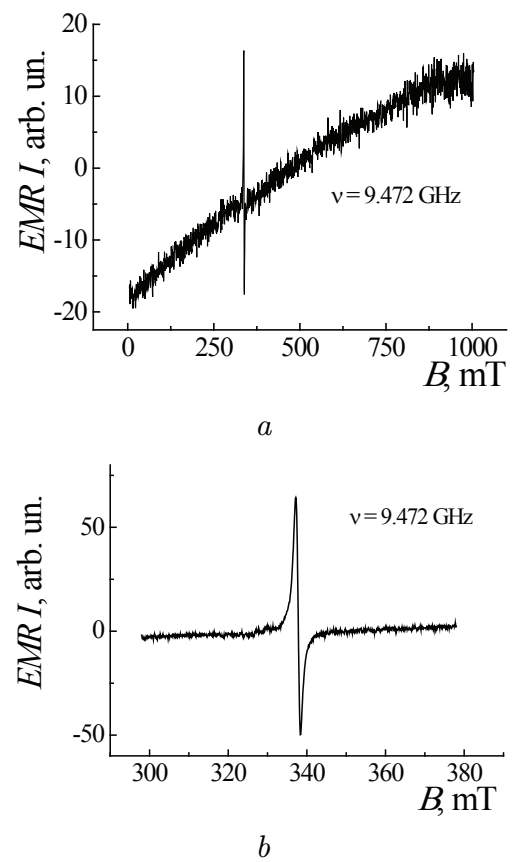

Fig. 3. RTEMR spectrum of a specimen functionalized with a mixture of sulfuric and nitric acids: full spectrum $(a)$ and the region in the vicinity of $g=2.00(b)$

imen, the quite intense narrow signal due to carbon inherited defects is observed (Fig. 4, b): $g=$ $=2.003 \pm 0.001$ and the line width $\Delta \mathrm{H}_{p p}=1.2 \pm$ $\pm 0.1 \mathrm{mT}$. Thus, the given specimen is more defective in the graphite layer plane in comparison with specimen No. 1. This correlates with the data of electron microscopic studies.

Figure 4 presents the general view of RTEMR spectra for specimens obtained by the sonication of TEG in ethanol (No. 5) (a) and acetone (No. 6) (b).

As is seen from Figures for both specimens, the weak signal due to carbon inherited defects is observed. 


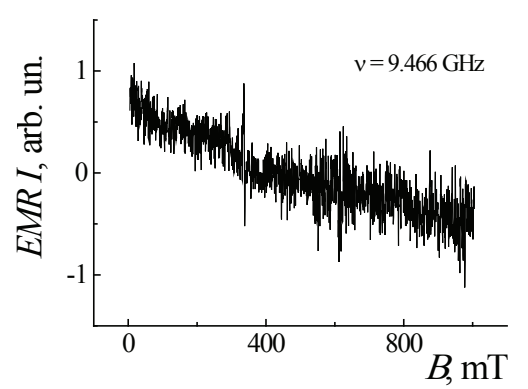

$a$

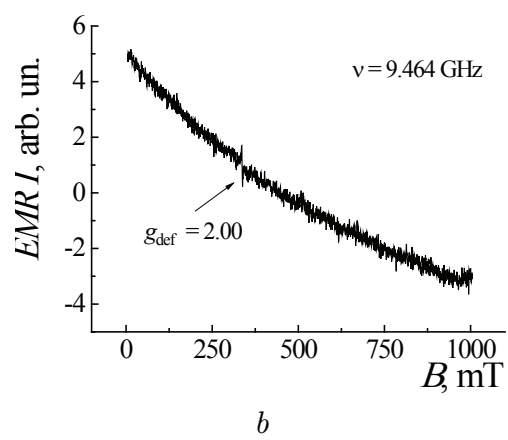

Fig. 4. RTEMR spectra of specimens No. $5(a)$ and No. $6(b)$

Thus, according to RTEMR studies, the GLSs obtained by the chemical treatment of TEG with a mixture of acids are most defective.

In order to reveal the peculiarities of the nanocarbon structure changes upon chemical and ultrasonic treatments, the obtained specimens of the GLSs were studied by the Raman spectroscopy method. To estimate the degree of uniformity of the structure of GLSs obtained with different methods, the Raman spectra were obtained from several points for each specimen of GLSs.

As is known, Raman spectroscopy is a powerful nondestructive method for the characterization of carbon-based materials such as carbon nanotubes, graphene, graphene-like structure, and graphites with different degrees of structure perfection [13-17]. There is only one intense band in the spectrum of monocrystalline ideal graphite. This is the so-called $G$-band $\left(\nu^{-1}=1580 \mathrm{~cm}^{-1}\right.$ and the half-width $\Delta \nu=$ $\left.=12.5 \pm 0.5 \mathrm{~cm}^{-1}\right)$ [13]. In the Raman spectra of less ordered graphites, an additional line appears in the region $\sim 1350 \mathrm{~cm}^{-1}$. This band ( $D$-band) is associated with the violation of selection rules due to a disorder, finite size of crystallites, defects, and rehybridization of carbon atoms from the $\mathrm{sp}^{2}-$ to $\mathrm{sp}^{3}$ - state. The intensity of the $D$-band increases with the defectiveness of a graphite structure. Herewith, the position of the $G$-band line is shifted from $1570 \mathrm{~cm}^{-1}$ to $1585 \mathrm{~cm}^{-1}$, and its halfwidth increases to $25 \mathrm{~cm}^{-1}$ [13]. With an increase in the $D$-band intensity, the $D^{\prime}$-band appears, often as the shoulder of the $G$-band. Raman spectroscopy also is used to identify graphene and graphene-like structures. With a decrease in the number of graphite layers in the graphene-like structure, the $G$-band is shifted to the short-wave region. Raman spectroscopic studies of graphene also have revealed very interesting phenomena. For example, the single sharp second-order Raman $G^{\prime}$-band has been widely used as a simple efficient way to confirm the presence of single-layer graphene [16]. The $G^{\prime}$-band of multilayer graphene can be fitted with multiple peaks due to the splitting of the electronic band structure of a multilayer material [16].

Figure 5 presents the typical Raman spectra for source TEG (Fig. 5, a) and specimens of GLSs (Figs. 5, b-g), and Table 2 presents the data of the profile analysis of the Raman spectra of the source TEG and obtained GLSs: center-of-gravity positions of the $D$ - and $G$-bands, half-width of the $G$-band, the ratio of integral intensities $A_{G} / A_{D}$, position of the $D^{\prime}$-band, positions of the overtones of the $G^{\prime}$-band $\left(G_{1}^{\prime}\right.$ and $\left.G_{2}^{\prime}\right)$, and ratio of their integral intensities $A_{G^{\prime} 1} / A_{G^{\prime} 2}$.

As is clearly seen from the figures, the general views of Raman spectra for all specimens are similar. There are the intense $G$-line assigned to the in-plane vibrations of the $\mathrm{C}-\mathrm{C}$ bond, weakly intense $D$-line activated by the presence of a disorder in the carbon systems and overtones, and $G^{\prime}$-line for all specimens, but the exact position of each line, the shapes of lines, as well as the ratios of the integral intensities of the lines for each specimen are significantly different.

Let us consider the more detailed spectra for each of the studied specimens.

For source TEG, the intense $G$-line is observed, whereas the $D$-line intensity is very small. The ratio of integral intensities $A_{G} / A_{D}$ is $\sim 0.11$ for all points of a specimen, which indicates a small defectness and a high uniformity of the specimen structure. The half-width of the $G$-line is $15 \mathrm{~cm}^{-1}$ that is close to the $G$-line half-width for high oriented pyrolytic graphite. The Raman spectrum also exhibits the band around $2700 \mathrm{~cm}^{-1}$ called the $G^{\prime}$-band and 

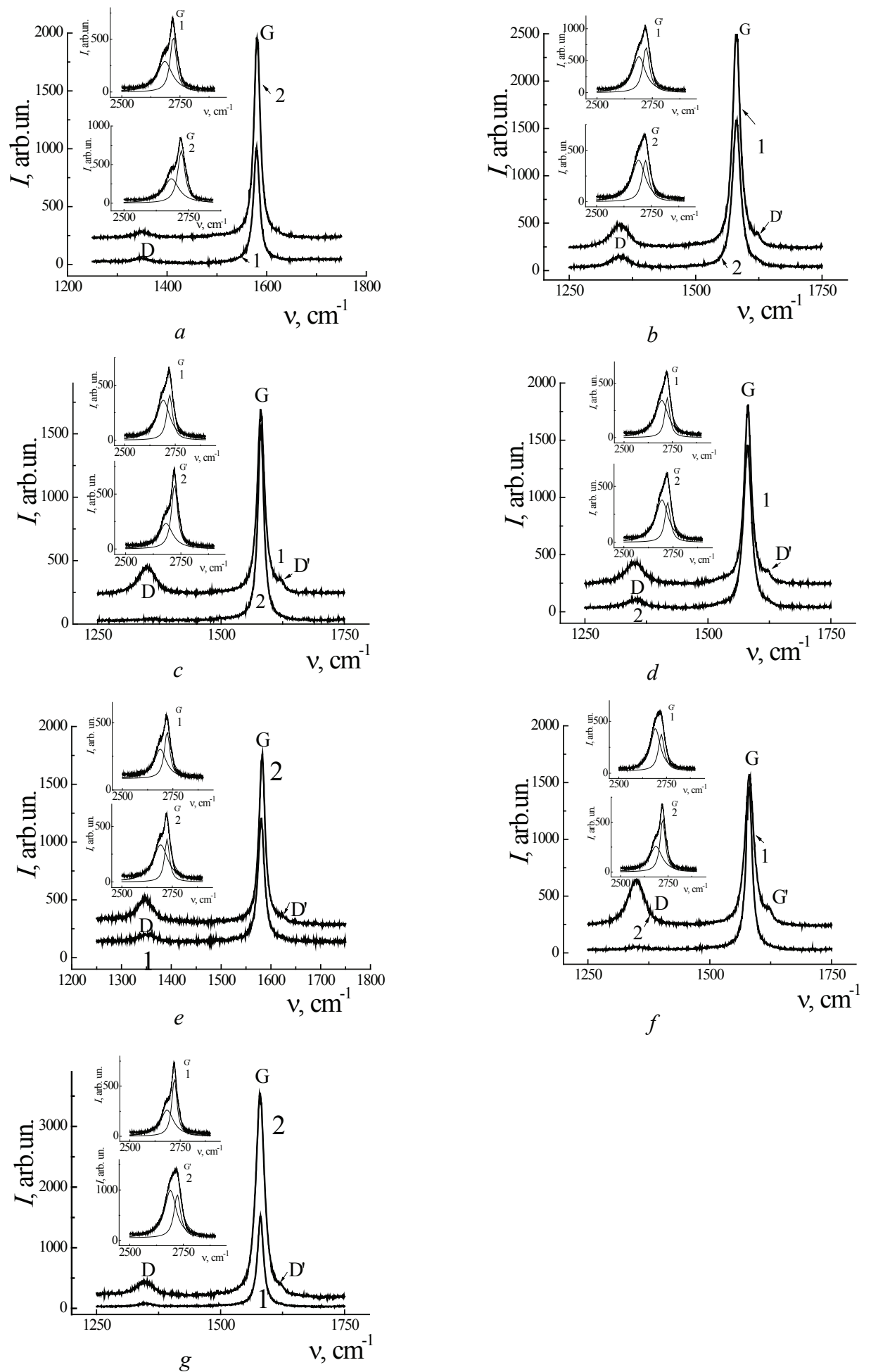

Fig. 5. The fragments of Raman spectra for source TEG $(a)$ and for specimens of GLSs: No. 1 $(b)$, No. $3(c)$, No. $4(d)$, No. $5(e)$, No. $6(f)$, No. $7(g)$ 
Table 2. The profile analysis of the Raman spectra of the source TEG and GLSs specimens

\begin{tabular}{|c|c|c|c|c|c|c|c|c|c|}
\hline \multicolumn{2}{|c|}{ No. } & $x_{D}, \mathrm{~cm}^{-1}$ & $x_{G}, \mathrm{~cm}^{-1}$ & $w_{G}, \mathrm{~cm}^{-1}$ & $x_{D^{\prime}}, \mathrm{cm}^{-1}$ & $A_{G} / A_{D}$ & $x_{G^{\prime} 1}, \mathrm{~cm}^{-1}$ & $x_{G^{\prime} 2}, \mathrm{~cm}^{-1}$ & $A_{G^{\prime} 1} / A_{G^{\prime} 2}$ \\
\hline \multirow[t]{2}{*}{ TEG } & 1 & 1347 & 1579.6 & 15.11 & - & 11.11 & 2683 & 2722 & 1.33 \\
\hline & 2 & 1351 & 1580.0 & 14.31 & - & 11.11 & 2685 & 2721 & 0.98 \\
\hline \multirow[t]{2}{*}{ No. 1} & 1 & 1350 & 1581.0 & 18.12 & 1623 & 4.76 & 2689 & 2721 & 1.53 \\
\hline & 2 & 1350.4 & 1580.7 & 18.94 & 1622 & 6.70 & 2692 & 2723 & 1.98 \\
\hline \multirow[t]{2}{*}{ No. 3} & 1 & 1349.8 & 1580.8 & 17.47 & 1622 & 4.76 & 2691 & 2722 & 2.05 \\
\hline & 2 & 1356.3 & 1580.7 & 14.09 & - & 11.11 & 2683 & 2721 & 0.98 \\
\hline \multirow[t]{2}{*}{ No. 4} & 1 & 1350 & 1581.1 & 17.5 & 16.23 & 2.86 & 2696 & 2723 & 2.48 \\
\hline & 2 & 1351 & 1580.5 & 19 & 1622 & 4.54 & 2694 & 2722 & 2.47 \\
\hline \multirow[t]{2}{*}{ No. 5} & 1 & 1346 & 1582.2 & 14.6 & 1623 & 3.12 & 2692 & 2724 & 2.62 \\
\hline & 2 & 1349 & 1580.5 & 15.4 & - & 5.55 & 2687 & 2721 & 1.36 \\
\hline \multirow[t]{2}{*}{ No. 6} & 1 & 1348.9 & 1581.3 & 21.89 & 1622 & 1.64 & 2692 & 2723 & 1.98 \\
\hline & 2 & 1353.8 & 1580.5 & 15.03 & - & 11.11 & 2686 & 2722 & 1.17 \\
\hline \multirow[t]{2}{*}{ No. 7} & 1 & 1349 & 1580.4 & 14.6 & - & 9.03 & 2685 & 2721 & 1.14 \\
\hline & 2 & 1347 & 1579.8 & 20.49 & 1622 & 5.5 & 2690 & 2722 & 1.95 \\
\hline
\end{tabular}

attributed to the overtone of the $D$-band. This band is essentially asymmetric for all specimens and is a combination of two lines $\left(G_{1}^{\prime}\right.$ and $\left.G_{2}^{\prime}\right)$. The exact position of the $G_{1}^{\prime}$ line is $2684 \mathrm{~cm}^{-1}$. The presence of an intense second line $\left(x_{G^{\prime} 2}=2722 \mathrm{~cm}^{-1}\right.$, $A_{G^{\prime} 1} / A_{G^{\prime} 2} \sim 1$ ) points out on a multilayer structure of TEG.

For the specimen obtained by the treatment of source TEG with a $\mathrm{KMnO}_{4}$ solution in sulphuric acid (specimen No. 1, Fig. 5, b) the ratio of integral intensities $A_{G} / A_{D}$ is essentially decreased in comparison with source TEG ( $\sim 4.76$ for the first point and $\sim 6.7$ for the second one). Such increase in the $D$-line intensity with respect to the $G$-line reflects a significant increase in the graphite layer structure defectness and a possible re-hybridization of carbon atoms. The some extension of the $G$-line (up to $18 \mathrm{~cm}^{-1}$ ) in comparison with source TEG also indicates the growth of the structure defectness. The $G$-line is somewhat shifted to the long wave region, that is a result of the reduction in the number of layers in the GLSs obtained with method No. 1. The shape of a broad $G^{\prime}$-band also significantly differs from the shape of the corresponding line for source TEG. The position of the first from two lines forming a broad $G^{\prime}$-band essentially shifts to the right. The ratio of the integral intensities of the lines $A_{G^{\prime} 1} / A_{G^{\prime} 2}$ increases up to 2 . Thus, all the data from the Raman spectra for the specimen obtained by the treatment of source TEG with a $\mathrm{KMnO}_{4}$ solution in sulphuric acid unambiguously point out on a decrease in the number of layers in GLSs and an increase in the defectness of graphite layers. The similarity of the values of the parameters at different points of the specimen shows a sufficient uniformity of the specimen structure.

Based on the Raman spectral view, the structure of the two specimens (No. 3 and No. 4) is less homogeneous. So, for the specimens obtained with the treatment of source TEG with a mixture of ethanol and toluene (specimen No. 3, Fig. 5, c) for different points, the ratio of the integral intensities $A_{G} / A_{D}$ varies from 4.76 to 11.11 . The last value of $A_{G} / A_{D}$ is the same as for source TEG. Thus, in this specimen, there are practically defect-free parts of graphite planes with crystallite sizes, which are similar to those in source TEG. However, there are areas with significant defects. In addition, such differences are present in the values of the $G$-line extension. For the first specimen point, the $G$-line extension is $15 \mathrm{~cm}^{-1}$ that coincides with the extension of the $G$-line in source TEG. For another specimen point, this line is more broadened (up to $18 \mathrm{~cm}^{-1}$ ). The same significant differences are observed for $G^{\prime}$-lines obtained from different points of the specimens. For the $G^{\prime}$-line obtained from a specimen point with a significant defect, the ratio of integral intensities $A_{G^{\prime} 1} / A_{G^{\prime} 2}$ is 2 , and the $G_{1}^{\prime}$ line is substantially shifted as compared with source TEG up to a position of $2691 \mathrm{~cm}^{-1}$. The G'-line ob- 
tained from another specimen point has the form, which is similar to the $G^{\prime}$-line form of TEG. The ratio of the integral intensities $A_{G^{\prime} 1} / A_{G^{\prime} 2}$ at this specimen point is 0.98 , and the position of the $G_{1}^{\prime}$-line exactly corresponds to the position of this line in the Raman spectrum of source TEG $\left(2683 \mathrm{~cm}^{-1}\right)$. Thus, this specimen has a pronounced heterogeneity. In this specimen, there are areas with a smaller number of graphite layers and a significant amount of defects in comparison with source TEG, as well as areas with a structure of source TEG.

A more homogeneous structure according to Raman spectra is observed for the specimen obtained with the treatment of source TEG with ethanol (specimen No.4, Fig. 5, d). For this specimen, the ratios $A_{G} / A_{D}$ are 2.86 and 4.55 , correspondingly, for different specimen points. Both $G$-lines obtained from different points are somewhat shifted to the longwave range as compared to source TEG. This indicates a decrease in the number of graphite layers in GLSs. The positions of the $G_{1}^{\prime}$-lines are also identical in the Raman spectra obtained from different specimen points. $G_{1}^{\prime}$-lines for both Raman spectra are shifted to the right in comparison with source TEG $\left(2695 \mathrm{~cm}^{-1}\right)$. The ratios of integral intensities $A_{G^{\prime} 1} / A_{G^{\prime} 2}$ are equal for two different specimen points.

Thereby, the chemical treatment of TEG on the whole results in the formation of GLSs with a small number of significantly defective graphite layers. However, such characteristics of the structure as homogeneity and defectness are very sensitive to the type of a chemical reagent that was used for such treatment.

The following specimens were obtained by the ultrasonic dispersion of source TRG in a chemical medium.

A slightly larger deviation of the spectral parameters is observed for a GLS obtained by the sonication of TEG in ethanol (specimen No. 5, Fig. 5, e) compared to the GLSs obtained with the treatment of TEG by ethanol in a magnetic stirrer (No. 4). The $G$-line wavelengths are somewhat shifted $\left(1582 \mathrm{~cm}^{-1}\right)$ for both Raman spectra. The ratio of the integral intensities $A_{G} / A_{D}$ are 3.12 and 5.55 for different points of the specimen. The $G^{\prime}$-line is also double. The $G_{1}^{\prime}$-lines are shifted to the right in both Raman spectra. The ratios $A_{G^{\prime} 1} / A_{G^{\prime} 2}$ are 2.62 and 1.31 , correspondingly. In general, the structure parameters of this specimen are similar to those of spec- imen No. 4, but the former is somewhat more heterogeneous.

The heterogeneity of the structure of the specimen obtained with the ultrasonic dispersion in acetone (specimen No. 6, Fig. 5, $f$ ) is even more pronounced according to the Raman spectrum. The Raman spectra obtained for different parts of the specimen are very different. For some parts of the specimens, the Raman spectrum is similar to the Raman spectrum of source TEG. The ratio $A_{G} / A_{D}$ is 11.11 , as for source TEG. The positions of the G- and $G_{1}^{\prime}$-lines correspond to the positions of these lines in source TEG, and the value of ratio $\left(A_{G^{\prime} 1} / A_{G^{\prime} 2}=1.17\right)$ indicates a significant number of graphite layers in a platelet. For other parts of the specimen, the ratio $A_{G} / A_{D}$ is the smallest for all the investigated specimens, $A_{G} / A_{D}$ is 1.64. That is, the integral intensities of the $G$ - and $D$-lines are almost equal. Such increase in the intensity of the $D$-line arising from the activation of the first-order scattering process of $\mathrm{sp}^{3}$-carbon indicates a growth in the number of defects in graphite layers or reducing the size of the crystallites along the graphite planes. The $G_{1}^{\prime}$-line is essentially shifted to the right, and $A_{G^{\prime} 1} / A_{G^{\prime} 2}$ is 2 . These data indicate a very small number of graphite layers in GLSs. So, specimen No. 6 is the most heterogeneous from all investigated specimens. However, it contains individual particles with the smallest number of graphite layers in GLSs.

The specimen obtained with the ultrasonic treatment of source TEG in water (No. 7, Fig. 5, g) is quite homogeneous from Raman spectral data. The integral intensity ratios $A_{G} / A_{D}$ for different specimen points are 9.03 and 5.50. These values are slightly smaller than for source TEG, but they are bigger, than for other specimens. The positions of the $G$ and $G_{1}^{\prime}$-lines are shifted to high frequencies. The ratio $A_{G^{\prime} 1} / A_{G^{\prime} 2}$ is in range from 1.14 to 1.95 . Thus, this specimen is less defective than other investigated specimens, but the number of graphite layers in the GLS obtained with method No.7 is larger, than in the GLSs obtained with other methods.

\section{Conclusions}

As the carried out investigations showed, all methods allow one to obtain GLSs from source TEG. However, the number of layers in the GLS, the shape of individual particles, structural-morphological charac- 
teristics, and the homogeneity of the sizes of particles are determined by the chemical substance, in which the dispersion took place and by the method of dispersion of particles.

It is revealed that the treatment of source TEG with a strong oxidizer $\left(\mathrm{KMnO}_{4}\right.$ solution in sulfuric acid or ethanol) lets one to get the planar graphenelike sheets with a small number of graphite layers. The treatment of source TEG with less strong oxidizers leds to the formation of highly defective carbon structures or structures those are very heterogeneous in size and number of graphite layers.

The use of the sonication for a dispersion of source TEG results in the formation of GLSs with a strongly expressed defects in graphite layers. However, the use of the sonication for a dispersion allowed us to obtain the GLSs with a small number of graphite layers even for the dispersion in water. When the sonication of TEG is carried out in acetone and ethanol, the formed GLSs are not very uniform in size and the number of layers. But the sonication of source TEG in acetone leads to the formation of GLSs with the smallest number of graphite layers.

1. A.H. Castro Neto, F. Guinea, N.M.R. Peres, K.S. Novoselov, A.K. Geim. The electronic properties of graphene. Rev. Mod. Phys. 81, 109 (2009).

2. K.S. Novoselov, S.V. Morozov, T.M.G. Mohinddin, L.A. Ponomarenko, D.C. Elias, A.K. Geim. Electronic properties of graphene. Phys. Stat. Sol. B 244, 4106 (2007).

3. J. Scott Bunch,Y. Yaish, M. Brink, K. Bolotin, P. McEuen. Coulomb oscillations and Hall effect in quasi-2D graphite quantum dots. Nano Lett. 5, 287 (2005).

4. A.K. Geim. Graphene: Status and prospects. Science 324, 1530 (2009).

5. I.A. Ovid'ko. Mechanical properties of graphene. Rev. Adv. Mater. Sci. 34, 12 (2013).

6. A.R. Ranjbartoreh, B. Wang, X. Shen. The characterization of graphene paper for flexible electronics application. J. Appl. Phys. 109, 014306 (2011).

7. K.M.F. Shahil, A.A. Balandin. Thermal properties of graphene and multilayer graphene: Applications in thermal interface materials. Solid State Communications $\mathbf{1 5 2}$ (15), 1331 (2012).

8. J.R. Potts, D.R. Dreyer, C.W. Bielawski. Graphene-based polymer nanocomposites. Polymer 52 (1), 5 (2011).
9. O. Yakovenko, L. Matzui, L. Vovchenko, A. Trukhanov, I. Kazakevich, S. Trukhanov, Yu. Prylutskyy, U. Ritter. Magnetic anisotropy of the graphite nanoplatelet-epoxy and MWCNT-epoxy composites with aligned barium ferrite filler. J. Mat. Sci. 52 (9), 5345 (2017).

10. L. Vovchenko, Yu. Perets, I. Ovsiienko, L. Matzui, V. Oliynyk, V. Launetz. Shielding coatings based on carbonpolymer composites. Surf. Coat. Techn. 211, 196 (2012).

11. I. Ovsiienko, O. Lazarenko, L. Matzui, O. Brusilovets, F.Le Normand, A. Shames. Influence of chemical treatment on the microstructure of nanographite. Phys. Stat. Sol. A 211, 2665 (2014).

12. Yu.S. Perets, I.V. Ovsiienko, L.L. Vovchenko, O.A. Brusilovets, I.P. Pundyk. Characterization of nanodispersed graphite. Ukr. J. Phys. 57, 219 (2012).

13. S.S. Bukalov, L.A. Mihalitsyn, Ya.V. Zubavichus. Investigation of the structure and physico-chemical properties of carbon materials. Ross. Khim. Zh. 1 (1), 83 (2006).

14. L. Bokobza, J. Zhang. Raman spectroscopic characterization of multiwall carbon nanotubes and of composites. Express Polymer Lett. 6, 601 (2012).

15. M.S. Dresselhaus, G. Dresselhaus, R. Saitoc, A. Joriod. Raman spectroscopy of carbon nanotubes. Physics Reports 409, 47 (2005).

16. Zhenhua Ni, Yingying Wang, Ting Yu, Zexiang Shen. Raman spectroscopy and imaging of graphene. Nano Res. 1, 273 (2008).

17. S. Costa, E. Borowiak-Palen. Raman study on doped multiwalled carbon nanotubes. Acta Phys. Polonica A 116, 32 (2009).

Received 11.12.17

I. Овсієнко, Т. Ленъ, Л. Мачуй,

О. Лазаренко, Ф. Ле Норманд, А. Шеймес

ОСОБЛИВОСТІ МІКРОСТРУКТУРИ ХІМІЧНО ОТРИМАНИХ ГРАФЕНОПОДІБНИХ ЧАСТИНОК

$\mathrm{P}$ е $з$ ю м е

В роботі методами електронної мікроскопії, EMP та Раманівської спектроскопії досліджуються графеноподібні структури. Графеноподібні структури були отримані хімічною обробкою та ультразвуковим диспергуванням вихідного терморозширенного графіту в різних хімічних середовищах. В результаті досліджень були оцінені кількість графітових шарів в графеноподібних структурах, форма окремих частинок та їі структурно-морфологічні особливості, а також однорідність розмірів частинок графеноподібних структур, отриманих різними методами з використанням різних хімічних реагентів. 